\title{
15th IFCARS/SPIE/EuSoMII/ISCAS Joint Workshop on Surgical PACS and the Digital Operating Room
}

Chairmen: Osman M. Ratib, MD, PhD (CH), Heinz U. Lemke, PhD (D) 